\title{
Comment on "Lifshitz-Matsubara sum formula for the Casimir pressure between magnetic metallic mirrors"
}

\author{
G. L. Klimchitskaya and V. M. Mostepanenko \\ Central Astronomical Observatory at Pulkovo of the Russian Academy of Sciences, Saint Petersburg, 196140, Russia and \\ Institute of Physics, Nanotechnology and Telecommunications, \\ Peter the Great Saint Petersburg Polytechnic University, St.Petersburg, 195251, Russia
}

\begin{abstract}
Recently Guérout et al. [Phys. Rev. E 93, 022108 (2016)] advocated that the lossless plasma model has to be redefined as the limit of the Drude model when the relaxation parameter goes to zero. It was claimed that the previously used plasma model cannot correctly describe the Casimir pressure between two plates made of both nonmagnetic and magnetic metals and has to be replaced with the redefined one. We show that the suggested redefinition does not satisfy necessary physical requirements imposed on the dielectric permittivity. We also present a plausible explanation to the fact that the lossless plasma model describes the Casimir pressure correctly even though it does not match the optical and electrical properties of metals.
\end{abstract}

PACS numbers: 11.10.Wx, 05.40.-a, 42.50.-p, 78.20.-e

It is common knowledge that the experimental data of all precise experiments on measuring the Casimir force between both nonmagnetic and magnetic metallic surfaces exclude theoretical predictions of the Lifshitz theory if the low-frequency dielectric permittivity is described by the Drude model [1 [6]. The same data are found in good agreement with theory if the dielectric permittivity at low frequencies is described by the lossless plasma model [1 $[6]$. In both cases the contribution of bound (core) electrons to the dielectric permittivity $\varepsilon(\omega)$ is found using the tabulated optical data for the complex index of refraction [7]. Keeping in mind that the presence of ohmic losses in the dielectric response of metals to real electromagnetic fields of low frequencies is a well confirmed fact, the exclusion of the Drude model is often considered as puzzling.

Reference [8] discusses this puzzle and arrives at a conclusion that the lossless plasma model which is commonly used has to be redefined as the limit of the Drude model

$$
\chi_{\gamma}(\omega) \equiv \varepsilon_{\gamma}(\omega)-1=-\frac{\omega_{p}^{2}}{\omega(\omega+i \gamma)}
$$

when the relaxation parameter $\gamma$ goes to zero $\left(\omega_{p}\right.$ is the plasma frequency of the metal). According to Ref. [8], the resulting susceptibility of the plasma model is

$$
\chi_{\eta}(\omega) \equiv \lim _{\gamma \rightarrow 0} \chi_{\gamma}(\omega)=-\frac{\omega_{p}^{2}}{\omega^{2}}-i \pi \omega_{p}^{2} \delta^{\prime}(\omega),
$$

where $\delta^{\prime}(\omega)$ is a derivative of the Dirac $\delta$-function. This is different from the commonly used susceptibility of the plasma model [9]

$$
\chi_{0}(\omega)=-\frac{\omega_{p}^{2}}{\omega^{2}},
$$

which is obtained from the Drude model (11) by putting $\gamma=0$ from the outset. In other words, the Drude model (11) is a discontinuous function of $\gamma$ at the point $\gamma=0$, and Ref. [8] favors the definition of the plasma model by Eq. (2) over that of Eq. (3). As stated in Ref. [8], "The lossless plasma model, with susceptibility $\chi_{0}(\omega)$, does not match the optical and electrical properties of gold, and it cannot describe correctly the Casimir pressure between two metallic plates." According to Ref. [8], "the plasma model can only be considered as an effective model at high frequencies $\omega \gg \gamma$. Considered in this manner, it has to be defined as the limit of the Drude model when $\gamma \rightarrow 0$ ", i.e., by Eq. (2). It should be noted, however, that the redefined plasma model (2) introduced in Ref. [8] also does not match the optical and electrical properties of gold. As recognized in Ref. 8], the use of the redefined model (2) does not solve the Casimir puzzle. Reference [8] considers that an advantage of their approach is the absence of a discontinuity between the Casimir forces calculated using the Drude model (1) and the redefined plasma model (2).

Below we demonstrate that the suggested susceptibility (2) does not satisfy some necessary physical requirements obeyed by the standard dielectric susceptibility (3). We also confirm that the lossless plasma model (3) combined with the contribution of core electrons does describe correctly the Casimir pressure between two metallic plates even though it does not match the optical and electrical properties of gold. A plausible explanation for this fact is presented.

First and foremost we note that the definition of the plasma model as the limiting case of the Drude model (11) was used much earlier in Ref. [10] with the result

$$
\chi_{\eta}(\omega) \equiv \lim _{\gamma \rightarrow 0} \chi_{\gamma}(\omega)=-\frac{\omega_{p}^{2}}{\omega^{2}}+i \frac{\omega_{p}^{2}}{\omega} \pi \delta(\omega) .
$$

This made it possible to formally bring the plasma model in agreement with the standard Kramers-Kronig relation [11]

$$
\chi_{\eta}(i \xi)=\frac{1}{\pi} \int_{-\infty}^{\infty} \frac{\omega \operatorname{Im} \chi_{\eta}(\omega)}{\omega^{2}+\xi^{2}} d \omega .
$$

The relation (5), however, is derived for the functions $\chi_{\eta}(\omega)$ analytic in the upper half-plane of complex $\omega$ and 
regular at $\omega=0$ or having a pole of no higher than of the first order. Thus, Eq. (5) is not applicable to the plasma model (3). The generalized Kramers-Kronig relations valid for the plasma-like susceptibilities of the form [12]

$$
\chi(\omega)=-\frac{\omega_{p}^{2}}{\omega^{2}}+\sum_{j=1}^{K} \frac{f_{j}}{\omega_{j}^{2}-\omega^{2}-i g_{j} \omega},
$$

where $\omega_{j} \neq 0$ are the resonance frequencies of $K$ core electrons, $g_{j}$ are their relaxation frequencies, and $f_{j}$ are their oscillator strengths are derived in Ref. [13]. Here, the parameters of oscillators $\omega_{j}, g_{j}$, and $f_{j}$ are determined from the tabulated optical data for $\mathrm{Au}, \mathrm{Ni}$ or any other metal. The permittivity (6) matches the optical data at $\omega>2 \mathrm{eV}$ but, as well as the permittivity $\chi_{0}(\omega)$, does not match the optical data at lower $\omega$. The generalized Kramers-Kronig relations are given by

$$
\begin{aligned}
& \operatorname{Re} \chi(\omega)=\frac{1}{\pi} \mathrm{P} \int_{-\infty}^{\infty} \frac{\operatorname{Im} \chi(\xi)}{\xi-\omega} d \xi-\frac{\omega_{p}^{2}}{\omega^{2}} \\
& \operatorname{Im} \chi(\omega)=-\frac{1}{\pi} \mathrm{P} \int_{-\infty}^{\infty} \frac{\operatorname{Re} \chi(\xi)+1+\frac{\omega_{p}^{2}}{\xi^{2}}}{\xi-\omega} d \xi \\
& \chi(i \xi)=\frac{1}{\pi} \int_{-\infty}^{\infty} \frac{\omega \operatorname{Im} \chi(\omega)}{\omega^{2}+\xi^{2}} d \omega+\frac{\omega_{p}^{2}}{\xi^{2}}
\end{aligned}
$$

Both the susceptibilities (3) and (6) satisfy the generalized Kramers-Kronig relations (7) directly with no modification. Using the definition of the derivative of $\delta$ function in the framework of the theory of distributions [14], it is easily seen that

$$
\omega \delta^{\prime}(\omega)+\delta(\omega)=0,
$$

i.e., Eq. (2) used in Ref. [8] is equivalent to Eq. (4) used in Ref. [10] (see also Refs. 13, 15]).

In fact the functions (2) and (44) cannot be continued to the upper half-plane of complex $\omega$ in a consistent way because they contain either the delta function or its derivative. This is also the reason why the imaginary parts of the functions (2) and (4) cannot be obtained from their real parts by means of some dispersion relation. Thus, these functions do not satisfy the necessary physical conditions required from the dielectric susceptibility [11]. Eventually, the use of such functions as susceptibilities would result in a violation of the causality principle. This invalidates the main result of Ref. [8] on a derivation of the Lifshitz-Matsubara sum formula for the Casimir pressure between magnetic metallic mirrors using the dielectric susceptibility (2).

Now we comment on the statement that "The lossless plasma model, with susceptibility $\chi_{0}(\omega), \ldots$ cannot describe correctly the Casimir pressure between metallic plates." However, currently the situation is that the measurement data of all precise experiments with both nonmagnetic and magnetic metals [1 6$]$ are in agreement with theoretical predictions of the Lifshitz theory at more than $90 \%$ confidence level [15] if the conduction electrons are described by the plasma model (3) and core (bound) electrons by the optical data 7]. Effectively this means that the plasma-like dielectric permittivity (6) is used. In experiments of Refs. [1-6] the difference in theoretical predictions which include and neglect the relaxation properties of free electrons is of only a few percent. Recently, however, one more, differential, force measurement has been performed between patterned magnetic metals [16, 17] based on the novel idea proposed in Refs. [18 20]. In the experiment of Refs. [16, 17] the theoretical predictions using the Drude model (1) and the plasma model (3) at low frequencies differ by the factor of many hundred. As a result, the theoretical prediction using the plasma model was confirmed, and the use of the Drude model was excluded conclusively.

The question arises on how this fact can be combined with the correct statement of Ref. [8] that "The lossless plasma model, with susceptibility $\chi_{0}(\omega)$, does not match the optical and electrical properties of gold,...", as opposed to "much better motivated lossy Drude model". We agree with the authors of Ref. [8] that this question remains to be solved. It is not true, however, that the very good agreement of the measurement data of many experiments with theoretical predictions using the plasma model (6) "correspond to inconsistent calculations" ... "which has to be corrected accordingly". The point is that the Casimir force originates from fluctuating electromagnetic field having a zero expectation value. As to the optical and electrical properties, they are measured as a response of gold, nickel and other metals to real electromagnetic fields with nonzero expectation values. The classical theory of electromagnetic fluctuations usually postulates similar reaction of a physical system to real and fluctuating fields [11]. Recent developments in theoretical and experimental investigation of the Casimir effect discussed above suggest that this might be not the case for some types of quantum fluctuations. This assumption is supported by the fact that the Lifshitz theory using the Drude model violates the Nernst heat theorem for both nonmagnetic and magnetic metallic test bodies with perfect crystal lattices $21-23]$. The redefined plasma model (2) and (4) also violates the Nernst heat theorem which is satisfied by the commonly used plasma model (3) and the generalized plasma model (6). Only future investigations will show whether the Casimir puzzle can be explained along these lines.

To conclude, the redefined plasma model (2) introduced in Ref. [8] is not only in disagreement with the optical and electrical properties of metals at low frequencies, similar to the plasma model (3), but it results in violation of thermodynamics and does not possess necessary physical properties required from dielectric susceptibilities, and, specifically, does not satisfy the KramersKronig relations. Thus, it cannot be considered as an alternative to the commonly used plasma model (3). 
[1] R. S. Decca, D. López, E. Fischbach, G. L. Klimchitskaya, D. E. Krause, and V. M. Mostepanenko, Ann. Phys. (N. Y.) 318, 37 (2005).

[2] R. S. Decca, D. López, E. Fischbach, G. L. Klimchitskaya, D. E. Krause, and V. M. Mostepanenko, Phys. Rev. D 75, 077101 (2007).

[3] R. S. Decca, D. López, E. Fischbach, G. L. Klimchitskaya, D. E. Krause, and V. M. Mostepanenko, Eur. Phys. J. C 51, 963 (2007).

[4] C.-C. Chang, A. A. Banishev, R. Castillo-Garza, G. L. Klimchitskaya, V. M. Mostepanenko, and U. Mohideen, Phys. Rev. B 85, 165443 (2012).

[5] A. A. Banishev, G. L. Klimchitskaya, V. M. Mostepanenko, and U. Mohideen, Phys. Rev. Lett. 110, 137401 (2013).

[6] A. A. Banishev, G. L. Klimchitskaya, V. M. Mostepanenko, and U. Mohideen, Phys. Rev. B 88, 155410 (2013).

[7] Handbook of Optical Constants of Solids, ed. E. D. Palik (Academic, New York, 1985).

[8] R. Guérout, A. Lambrecht, K. A. Milton, and S. Reynaud, Phys. Rev. E 93, 022108 (2016).

[9] E. M. Lifshitz and L. P. Pitaevskii, Physical Kinetics (Pergamon, Oxford, 1981).

[10] C. Kittel, Introduction to Solid State Physics (Wiley, New
York, 1996).

[11] L. D. Landau and E. M. Lifshitz, Statistical Physics, Part I (Pergamon, Oxford, 1980).

[12] G. L. Klimchitskaya, U. Mohideen, and V. M. Mostepanenko, Rev. Mod. Phys. 81, 1827 (2009).

[13] G. L. Klimchitskaya, U. Mohideen, and V. M. Mostepanenko, J. Phys. A: Math. Theor. 40, 339 (2007).

[14] V. S. Vladimirov, Equations of Mathematical Physics (Decker, New York, 1971).

[15] V. M. Mostepanenko, J. Phys.: Condens. Matter 27, 214013 (2015).

[16] G. Bimonte, D. López, and R. S. Decca, Phys. Rev. B 93, 184434 (2016).

[17] R. S. Decca, Int. J. Mod. Phys. A 31, 1641024 (2016).

[18] G. Bimonte, Phys. Rev. Lett. 112, 240401 (2014).

[19] G. Bimonte, Phys. Rev. Lett. 113, 240405 (2014).

[20] G. Bimonte, Phys. Rev. B 91, 205443 (2015).

[21] V. B. Bezerra, G. L. Klimchitskaya, V. M. Mostepanenko, and C. Romero, Phys. Rev. A 69, 022119 (2004).

[22] M. Bordag and I. Pirozhenko, Phys. Rev. D 82, 125016 (2010).

[23] G. L. Klimchitskaya and C. C. Korikov, Phys. Rev. A 91, 032119 (2015); 92, 029902(E) (2015). 